\title{
Bracing and reciprocation
}

\author{
J. C. Davenport, ' R. M. Basker, ${ }^{2}$ J. R. Heath, ${ }^{3}$ J. P. Ralph, ${ }^{4}$ P-O. Glantz, ${ }^{5}$ and P. Hammond, ${ }^{6}$
}

\author{
This article describes how bracing can be used \\ to produce stable RPDs which distribute forces \\ efficiently to the supporting tissues. The \\ contribution of reciprocation to effective \\ clasping is also discussed.
}

\author{
In this part, we will discuss \\ - Distribution of forces \\ - Bracing components \\ - Distal extension saddle problems \\ - Types of reciprocation \\ - Guide surfaces
}

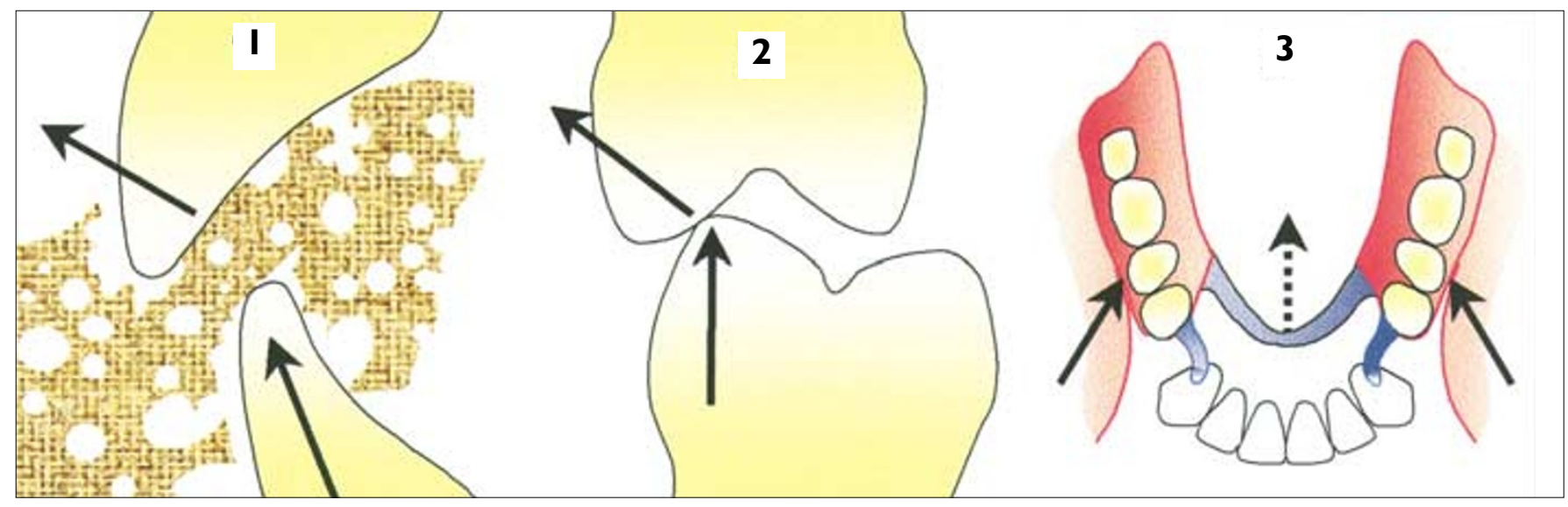

\section{Bracing}

Fig. I - Bracing

Horizontal forces are generated during function by occlusal contact ( $I$ and 2 ) and by the oral musculature surrounding the denture (3). These forces tend to displace the denture in both antero-posterior and lateral directions.

\footnotetext{
$1^{*}$ Emeritus Professor, University of Birmingham, UK; ${ }^{2}$ Professor of Dental Prosthetics, University of Leeds and Consultant in Restorative Dentistry, Leeds Teaching Hospitals NHS Trust, Leeds, UK; ${ }^{3}$ Honorary Research Fellow, University of Manchester (Formerly Senior Lecturer in Restorative Dentistry, University of Manchester) and Consultant in Restorative Dentistry, Central Manchester Healthcare Trust, Manchester, UK; ${ }^{4}$ Consultant in Restorative Dentistry, Leeds Teaching Hospitals NHS Trust and Senior Clinical Lecturer, University of Leeds and Honorary Visiting Professor, Centre for Dental Services Studies, University of York, York, UK; ${ }^{5}$ Professor of Prosthetic Dentistry, Consultant in Prosthetic Dentistry, Faculty of Odontology, University of Malmo, Sweden; ${ }^{6}$ Professor of Informatics, Eastman Dental Institute for Oral Health Care Sciences, University College London ${ }^{*}$ Correspondence to: 5 Victoria Road, Harborne, Birmingham B17 OAG email: john.davenport@btclick.com REFEREED PAPER

(C) British Dental Journal 2001; 190: 10-14
}

\section{New publications:}

All the parts which comprise this series (which will be published in the BDJ) have been included (together with a number of unpublished parts) in the books A Clinical Guide to Removable Partial Dentures (ISBN 0-904588-599) and A Clinical Guide to Removable Partial Denture Design (ISBN 0-904588-637). Available from Macmillan on 01256302699

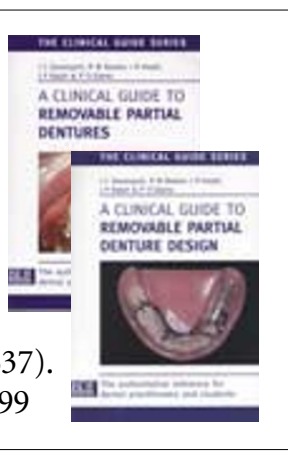



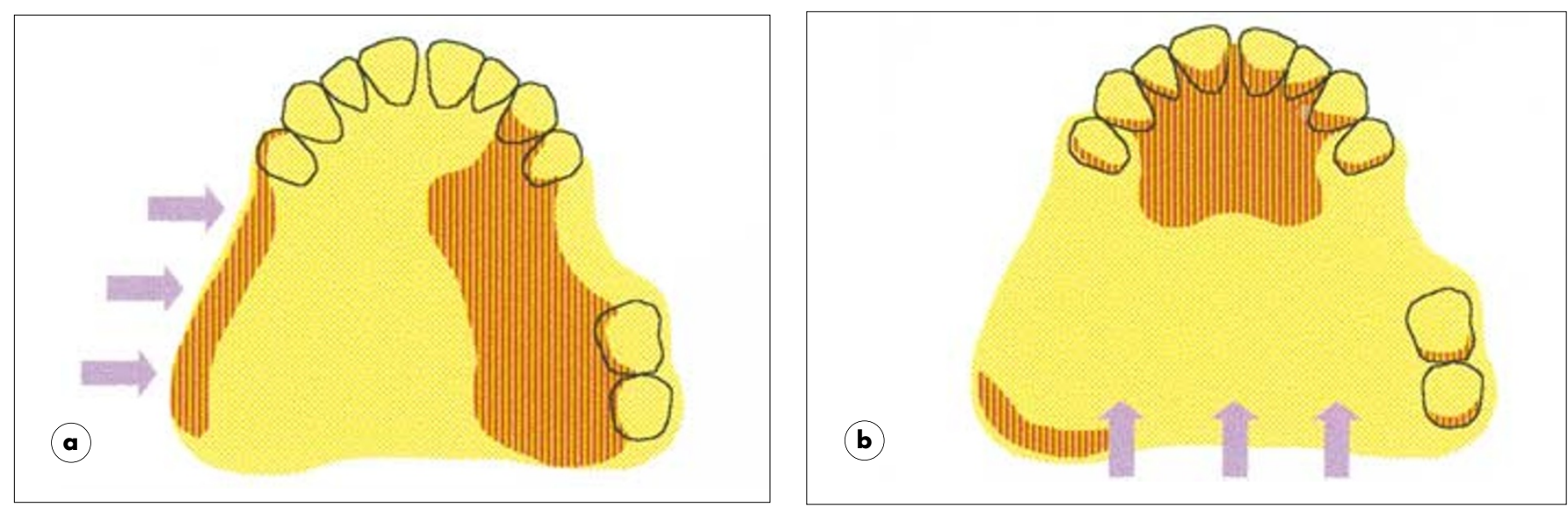

Fig. 2a and $b-$ Bracing

The horizontal forces are resisted by placing rigid components of the denture (bracing components) against suitable vertical surfaces on the teeth and residual ridges. Parts of a denture resting against the stippled areas will resist the forces whose directions are shown by the arrows. It is important to appreciate that bracing occurs only when the denture is fully seated.

Fig. 3 - Bracing

The lateral forces in particular are capable of inflicting considerable damage on the periodontal tissues and alveolar bone in the edentulous areas. Thus they have to be carefully controlled. Bracing on teeth may be achieved by means of rigid portions of clasp arms (1) or plates (2). Bracing on the ridges and in the palate is obtained by means of major connectors and flanges (3).

A distal extension saddle creates particular problems, as it is capable of being displaced posteriorly and of rotating in the horizontal plane. Furthermore, the lateral force must be distributed

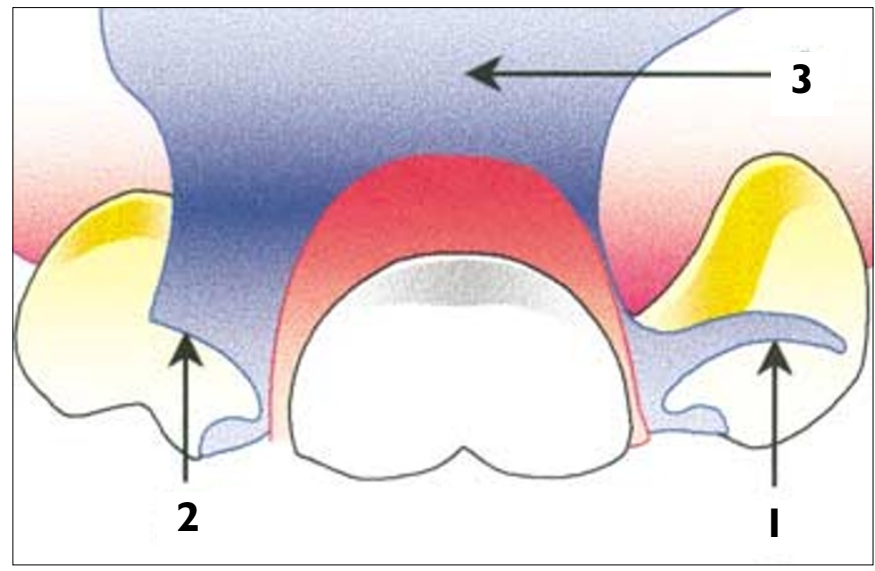

widely so that tissue damage is avoided. The problems are more acute in the mandibular arch.

\section{Fig. 4 - Bracing}

Those components of the RPD coloured blue are capable of resisting lateral forces coming from the direction indicated by the arrows. Needless to say, lateral forces in the opposite direction will be resisted by the mirror images of these components.

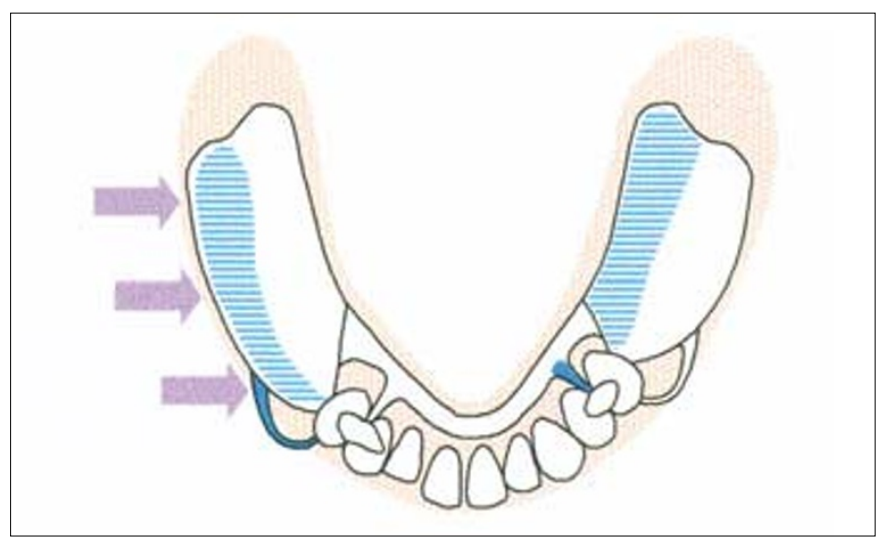




\section{PRACTICE prosthetics}
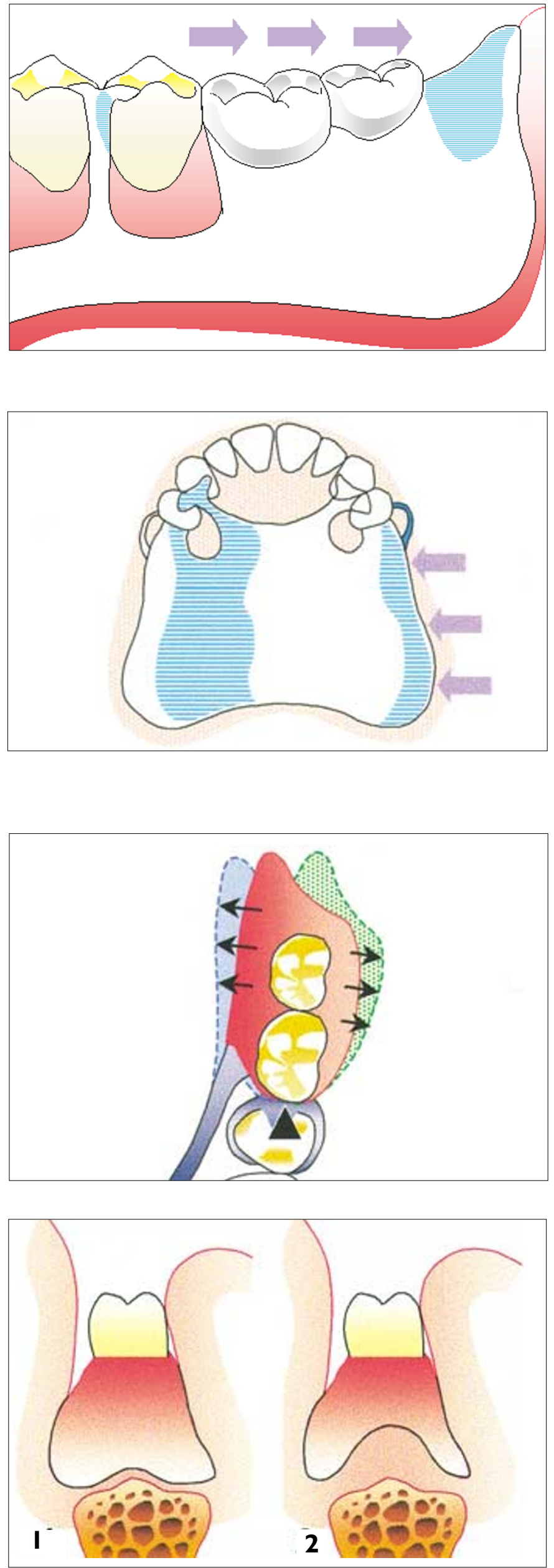

Fig. 5 - Bracing

Posterior movement of the distal extension saddle is prevented by coverage of the pear-shaped pad and by the minor connector which contacts the mesiolingual surface of the premolar tooth.

\section{Fig. 6-Bracing}

Effective distribution of the lateral force in the maxilla is less of a problem as much of it can be transmitted to the bone of the palatal vault by extensive palatal coverage. Those components of the RPD coloured blue are capable of resisting lateral forces coming from the direction indicated by the arrows.

\section{Fig. 7 - Bracing}

The posterior part of the distal extension saddle is capable of rotating in the horizontal plane. If a long saddle is clasped rigidly to a single abutment tooth the rotatory movement can transmit considerable force to that tooth.

\section{Fig. 8-Bracing}

The flatter the ridge ( 1 ) or the more compressible the mucosa (2), the greater is the potential for movement. It should also be remembered that the close fit of a denture will deteriorate following resorption of the residual ridge. Once more the potential for rotatory movement is increased. 


\section{Fig. 9 - Bracing}

Rotation can be resisted effectively by this design that incorporates appropriately placed bracing elements and joins them with a rigid connector. Rotation of the right saddle in the direction of the blue arrow is resisted by the minor connector contacting the mesial surface of LL5 (35). Movement of the saddle in the direction of the red arrow will be resisted by the minor connector contacting the distal surface of the same tooth.

\section{Fig. 10-Bracing}

Rotation and anteroposterior movement of bounded saddles are resisted by contact of the saddles with the abutment teeth. It therefore remains to design bracing elements which will safely distribute the lateral forces acting on the denture. The bracing elements that oppose a lateral force indicated by the arrows are shown in this illustration.

\section{Fig. I I - Bracing}

(I) Anterior displacement of a maxillary Kennedy Class IV denture can be resisted by elements of the framework contacting the distopalatal and disto-buccal surfaces of the teeth and, in some cases, by the connector covering the anterior slope of the palate.

(2) Posterior displacement is resisted by the labial flange, by contact between the saddle and the mesial surfaces of UR2 (I2) and UL3 (23), by contact of the minor connectors against the mesiopalatal surfaces of UR7 (17) and UL7 (27), and by the mesio-palatal and mesio-buccal portions of the clasp arms on UR6 (16), UR7 (17), UL6 (26) and UL7 (27).
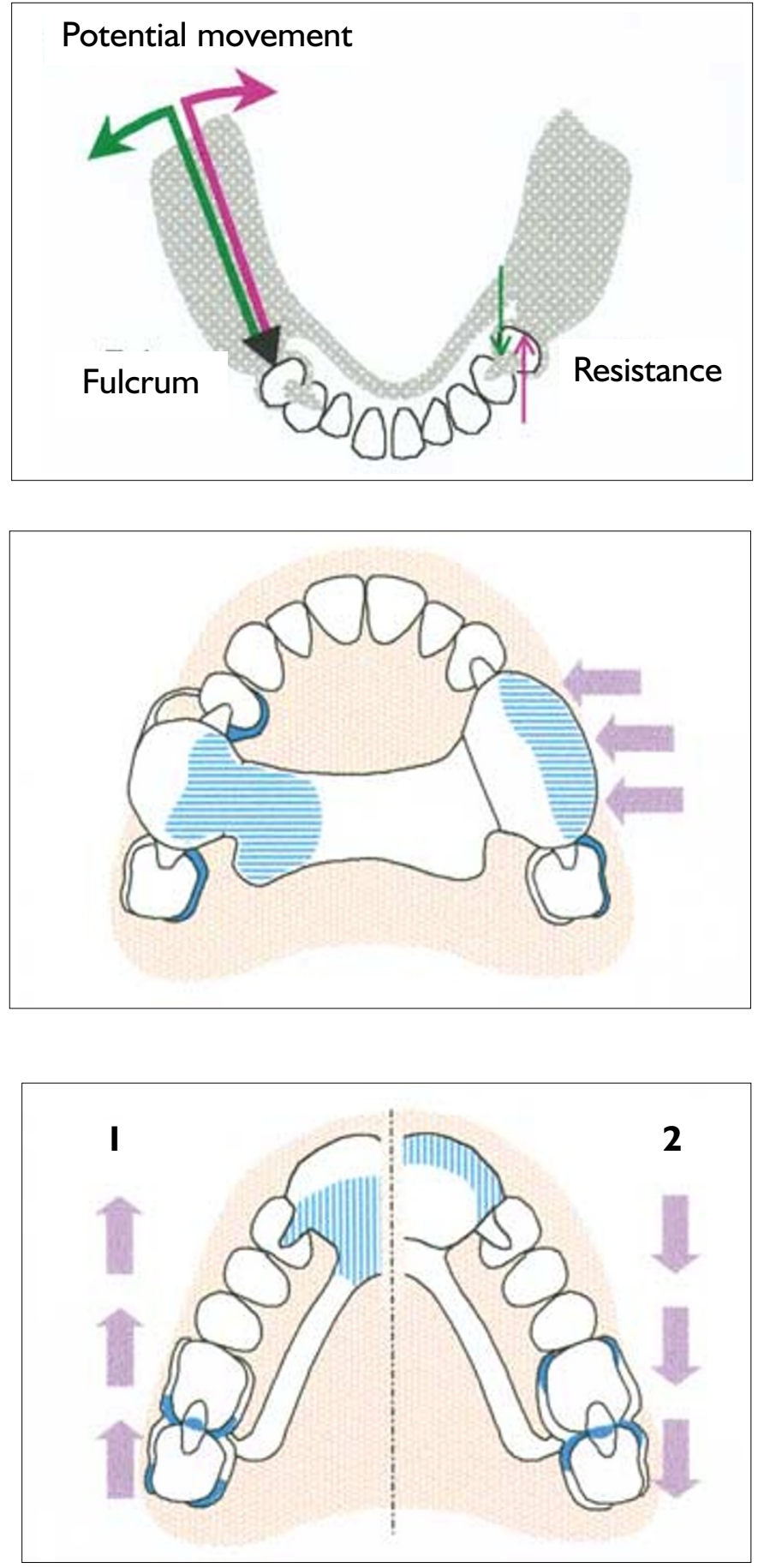

\section{Reciprocation}

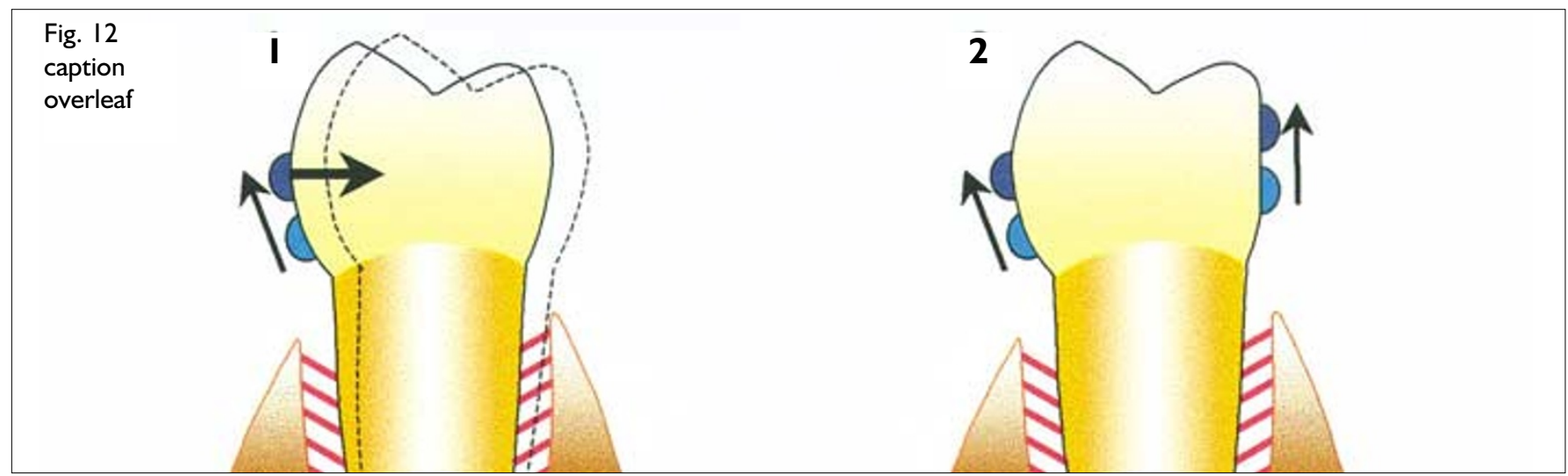




\section{PRACTICE prosthetics}

\section{Fig. 12 - Reciprocation}

The bracing element which is in contact with the side of the tooth opposite the retentive clasp can also play an important role in the effectiveness of the latter, and thus in the overall retention of the denture. (I) A horizontally directed force is produced as a retentive arm is displaced in an occlusal direction over the bulbosity of a tooth. If the clasp arm is unopposed the tooth is displaced in the periodontal space and much of the retentive capability will be lost. (2) If the retentive clasp is opposed by a rigid component which maintains contact with the tooth as the retentive arm moves over the bulbosity of the tooth, displacement of the tooth is resisted, the retentive arm is forced to flex and thus the efficiency of the retentive element is increased. This principle is known as reciprocation. It is thus apparent that reciprocation is required as the denture is being displaced occlusally whilst the bracing function, as mentioned earlier, comes into play when the denture is fully seated.

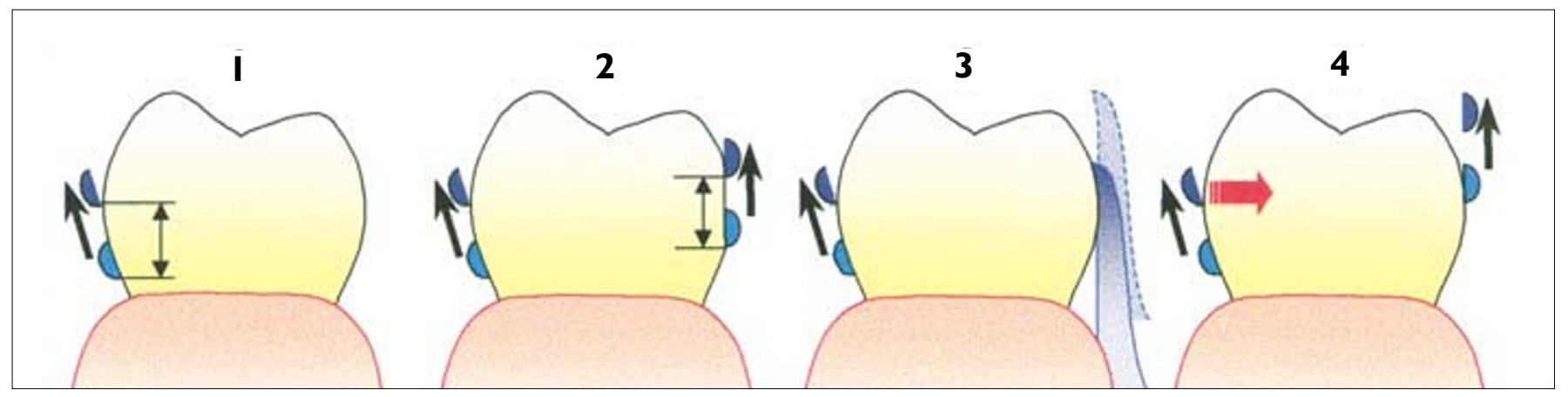

Fig. 13 - Reciprocation

(I) A clasp is effective in retention from its position when the denture is fully seated to where it escapes over the bulbosity of the tooth. This vertical measurement may be termed the 'retention distance'. It will be appreciated that the reciprocal element on the other side of the tooth should be in continuous contact with the tooth surface as the retentive arm traverses the 'retention distance'. Effective reciprocation can be achieved either (2) by a clasp arm contacting a guide surface of similar height to the 'retention distance', or (3) by a plate making continuous contact with the tooth surface as the retentive arm moves through its 'retention distance'. (4) If the reciprocating clasp is placed on a tooth without an adequate guide surface, it will lose contact with the tooth before the retentive arm has passed over the maximum bulbosity of the tooth and fail to provide effective reciprocation.
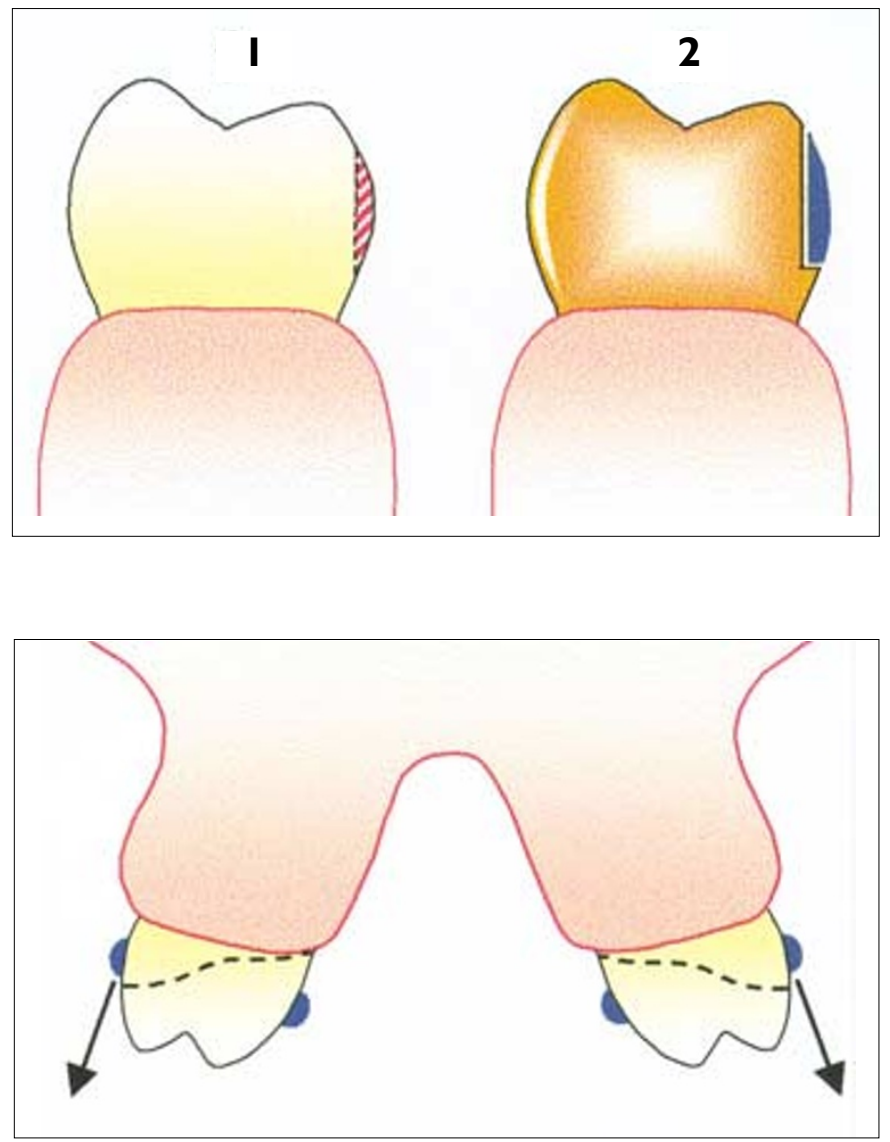

Fig. 14-Reciprocation

On rare occasions it may be possible to find a guide surface which occurs naturally on a tooth. More often it will be necessary to create a suitable surface by (I) minimal shaping of the enamel or (2) building the appropriate surface into a cast metal restoration, always supposing that such an extensive restoration is justified on that particular tooth.

\section{Fig. 15 - Reciprocation}

If the tooth surface on which the bracing arm is to be placed has a survey line at the level of the gingival margin, it will not be possible to achieve effective reciprocation on the same tooth. In such circumstances one may use the principle of cross-arch reciprocation, where a retentive clasp on one side of the arch opposes a similar component on the other side. The retentive clasps can be placed either buccal/buccal (as in the illustration) or lingual/lingual. The disadvantage of this approach is that, as the bracing arms leave the tooth surfaces, the teeth will move in their sockets. This 'jiggling' action is potentially damaging to the supporting tissues and will reduce the effectiveness of the retention. 